\title{
2 A helical charge simulation based 3-D method for 3 calculating AC stranded conductor's corona loss in 4 the corona cage
}

Yunpeng Liu' ${ }^{1}$, Shilong Huang ${ }^{*}$, Shugang Liu ${ }^{1}$, Daran Liu ${ }^{1}$, and Zhicheng Huang ${ }^{1}$

1 Hebei Provincial Key Laboratory of Power Transmission Equipment Security Defense, North China Electric Power University, No.619 North of Yonghua Street, Baoding 071003, China; liuyunpen@ncepu.edu.cn(Y.L.); liushugang@ncepu.edu.cn(S.L.); 771276676@qq.com(D.L.); 648277489@qq.com(Z.H.)

* Correspondence: simonhuang@ncepu.edu.cn; Tel.: +86-151-0020-8295

Abstract: Corona loss generated from conductors is one of the important design factors on ac transmission lines. Based on helical charge simulation method, the 3-D calculation model for corona loss considering the conductors' outer strands in the corona cage is established. With regard to 2-D corona-loss calculation method, the calculation model proposed in this paper can take the electric field strength's nonuniformity along the axial direction of stranded conductor into consideration, furthermore, it can also calculate the corona loss when the conductor has sag. The calculation results of the LGJ-300/40 and LGJ-400/35 in the small corona cage well coincide with the measurement results, which indicate that the corona-loss calculation model is appropriate. In addition, the corona loss of the bundle conductors $4 \times$ LGJ720 with sag in UHV corona cage is also analyzed.

Keywords: corona loss, helical charge, 3-D calculation model, stranded conductor, sag

\section{Introduction}

In alternating current (AC) lines, when the field strength around transmission line exceeds the breakdown electric field strength of air, the air near the conductor is ionized, which is called corona discharge. The corona discharge results in the production of different space charge (positive ions, negative ions and electrons), the charge carriers showing different polarity with the conductor are attracted to the conductor and may lose their charge on the conductor surface while those with similar polarity with the conductor are pushed away from it towards the ground. The energy loss caused by such back-and-forth movement of space charge in power cycle is called corona loss. Corona loss, which has been researched for many years, shows important significances for conductors selection, and is a considerable criterion for measuring the operation economy of transmission lines [1-4].

Up to now, AC conductors' corona loss has been extensively studied through experiments using small indoor corona cage, large outdoor corona cage and test lines. The test results show that these factors including conductor radius, bundle number, bundle spacing, distance to ground and surface roughness coefficients can influence corona loss [5-12]. 
When AC voltage is applied, the space line charge will go back and forth at power frequency, and it's more difficult to calculate ac ion-flow field, therefore, there is little research on the calculation model of AC conductors' corona loss. Clade, Abdel-Salam, Li, Liu researched the calculation model. Clade calculated the corona loss of single conductor in corona cage based on Kaptzov and Deutch assumptions while ignoring the influences of space charges on the electric field direction[13-15]. By abandoning the Deutsch assumption, Abdel-Salam researched the single, triple and multi phases transmission-line corona loss, but he did not consider the inhomogeneity of electric field on the conductor surface and believed that charges on the conductor surface are emitted uniformly [16,17]. By taking charge emission's inhomogeneity on the conductors surface into consideration, Li researched the ion flow of multiphase and bundle conductors [18]. Liu adopted the Li's improved calculation model and researched the corona loss of single and bundle conductors in the corona cage $[19,20]$.

However, the present corona loss calculation models are all for 2-D models, which assume that the conductor has infinite length as well as smooth and straight structure, ingoring the electric-field strength's nonuniformity along the axial direction of stranded conductor. Due to the finite length and conductors' external twisting structure, the electric field strength on the conductor surface were uneven. Meanwhile, the 2-D calculation model can't take the conductor's sag or wind lean into consideration either. so it is necessary to develop a 3-D calculation model for corona loss .

In this paper, simulation charges which have the same spiral form as the outer strand of the conductor are used to calculate corona loss of the conductor owing to spirally wounded outer strand of the conductor [21]. Every corona cage wall is equivalent to the ground plane and simulated by the helical charge as well. By utilizing the Kaptzov assumption and abandoning the Deutsch assumption, the processes including emission, transfer and recombination of space line charges are simulated considering the inhomogeneity of charge emission on the surface of conductor, and the 3-D calculation model for corona loss in the corona cage is established. The calculation model's accuracy is verified through test data in small corona cage and UHV corona cage.

\section{Calculation methods}

\subsection{Twisting form of the external of stranded conductors}

The conductors' outer strand is spirally winded on inner strand. If a point $M(x, y, z)$ in a space rotates about $x$ axis at the angular velocity $\omega$ on a cylindrical surface $y^{2}+z^{2}=R^{2}$, and rises along the positive direction parallel to $\mathrm{x}$ axis at the linear velocity $v$, the motion track of point $M$ is known as a helical line. When $\omega t=2 \pi$, the distance $h$ of point $M$ moving along the axial direction is called the pitch. As shown in Figure 1, the pitch length $L p=P F \times 2 R$ in engineering, $P F$ is the conductor's pitch factor and $R$ is outer diameter. Parameters of two common conductors are shown in Table 1 [23]. Hence, the location of point $M$ which is related to the $L_{p}$ and the conductor radius $R$ can be obtained through (1)-(3).

$$
x=\frac{L_{p}}{2 \pi} \theta
$$


78

$$
z=R \cos \left(\frac{2 \pi x}{L_{p}}\right)
$$

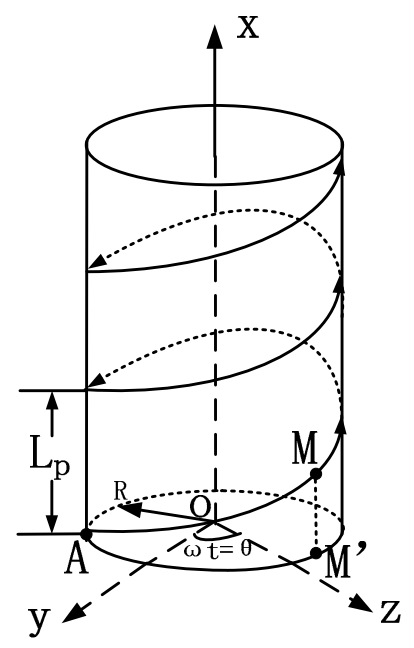

Figure 1. External twisting form of the stranded conductors

Table1. Parameters of two common stranded conductors

\begin{tabular}{|c|c|c|c|c|c|}
\hline \multirow[b]{2}{*}{ Conductor } & \multicolumn{2}{|c|}{ Aluminum strands } & \multirow{2}{*}{$\begin{array}{l}\text { the conductor } \\
\text { radius } R(\mathrm{~mm})\end{array}$} & \multirow{2}{*}{$\begin{array}{c}L_{p} \\
(\mathrm{~mm})\end{array}$} & \multirow[b]{2}{*}{$P F$} \\
\hline & $\begin{array}{l}\text { Number of outer } \\
\text { strands } n_{0}\end{array}$ & $\begin{array}{c}\text { Diameter of outer } \\
\text { strands } R_{g}(\mathrm{~mm})\end{array}$ & & & \\
\hline LGJ300-40 & 15 & 3.99 & 23.94 & 277 & $10-12$ \\
\hline LGJ400-35 & 22 & 3.22 & 26.82 & 300 & $10-12$ \\
\hline
\end{tabular}

\subsection{Helical charges}

The simulation helical charges are placed inside each strand of the external layer and take the same helical shape as the strand and repeat along $x$-axis after a pitch, assuming that conductor is finite long and segmented into finite number of pitches $(-k \ldots,-1,0,1, \ldots+k)$, in each pitch, any helical charge, $q_{h}$, is divided into $n$ finite line charges, having length $l$ and equal projections along the $x$-axis, as demonstrated in Figure 2. The number of simulation helical charges $q_{h}$ is assumed to be 3 times the number of strands in the outer layer $\left(3 \times n_{0}\right), n_{8}$ simulation helical charges are placed on corona cage walls, that is, the total number of simulation line charges with an equal pitch is $N=n \times(3 \times$ $\left.n_{0}+n_{g}\right)$. Owing to simulation line charges are repetitions of equal pitch along $x$ direction, the

91 unknown simulation line charges are only those in Pitcho, and the rest simulation line charges can be obtained through the coordinate transformation. 


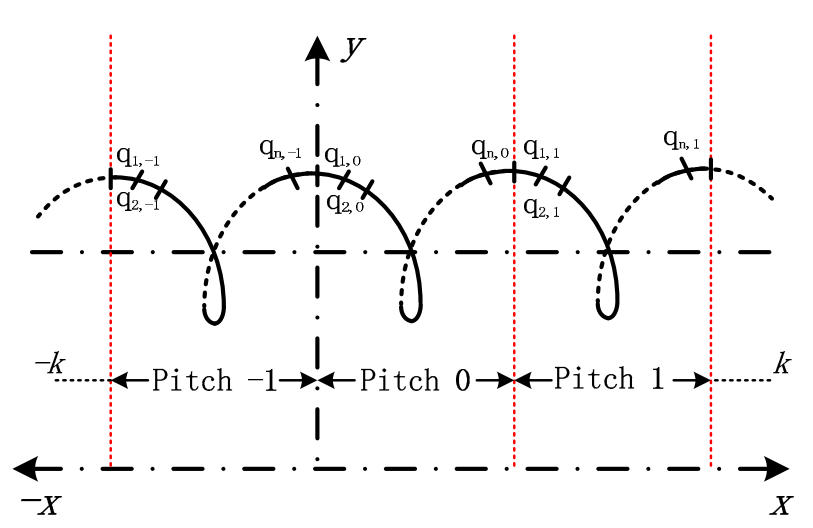

Figure2. Helical charges

\section{Coordinates of simulation line charges and boundary points.}

Figure 3 illustrates the cross section of LGJ400-35 which has $n_{0}=22$, an overall radius $R=13.41 \mathrm{~mm}$ in the corona cage. Where, the red ' $o$ ' and blue ' $x$ ' represent the arrangement points of simulation helical charges and boundary points, respectively. Inside each strand of the outer layer, the three helical simulation charge $q_{h 1}, q_{h 2}$ and $q_{h 3}$ are assumed to be spaced radially from the strand center with the distances of $f_{1} R_{g}, f_{2} R_{g}$ and $f_{3} R_{g}$, where, $R_{g}$ is the strand radius, $0<f_{2}=f_{3}<f_{1}<1$. $\Psi$ indicates the deviation angle of helical charges $q_{h 2}$ and $q_{h 3}$ to $q_{h 1}$. When a corona cage with a square section is equivalent to a cylindrical one, the equivalent diameter $R_{\text {cage }}=1.08 \times L, L$ is the cross-section dimension of the corona cage [4]. In order to verify whether the potential boundary conditions are met, the boundary points with the same number and deviation angle are chosen on the outer strands and corona cage surface corresponding to the each simulation line charge, hence, $N$ boundary points are chosen for Pitcho, and each boundary point lies on its locus at the middle of the corresponding simulation line charge. The sketch map of helical charges with Pitcho is shown in Figure 4.

Due to the simulation linear charges with charge $Q_{j}\left(Q_{j}=q_{j, 0, j}=1,2, \ldots, N\right)$, supposing that the starting coordinate is $A_{m}\left(x_{m}, y_{m}, z_{m}\right)$ and the length is $l_{j}$, the potential coefficient $P_{i, j}$ and field strength coefficients $f x_{i j}, f y_{i j}$ and $f z_{i j}$ at arbitrary point $A_{i}\left(x_{i}, y_{i}, z_{i}\right)$ are shown as follows:

$$
P_{i j}=\frac{1}{4 \pi \varepsilon_{0} l_{j}}\left[\ln \frac{\left(l_{j}-x_{1}\right)+\gamma}{\left(-x_{1}\right)+\delta}\right]
$$

$$
f x_{i j}=\frac{1}{4 \pi \varepsilon_{0} l_{j}}\left[\frac{1}{\gamma}-\frac{1}{\delta}\right]
$$

$$
f y_{i j}=\frac{1}{4 \pi \varepsilon_{0} l_{j}}\left[\frac{y_{1}}{\left(y_{1}\right)^{2}+\left(z_{1}\right)^{2}}\right]\left[\frac{\left(l_{j}-x_{1}\right)}{\gamma}+\frac{x_{1}}{\delta}\right]
$$

$$
f z_{i j}=\frac{1}{4 \pi \varepsilon_{0} l_{j}}\left[\frac{z_{1}}{\left(y_{1}\right)^{2}+\left(z_{1}\right)^{2}}\right]\left[\frac{\left(l_{j}-x_{1}\right)}{\gamma}+\frac{x_{1}}{\delta}\right]
$$

$$
\text { Where, }
$$


118

$$
\gamma=\sqrt{\left(l_{j}-x_{1}\right)^{2}+\left(y_{1}\right)^{2}+\left(z_{1}\right)^{2}}
$$

119

$$
\delta=\sqrt{x_{1}^{2}+\left(y_{1}\right)^{2}+\left(z_{1}\right)^{2}}
$$

120

$$
x_{1}=x_{i}-x_{m}
$$

121

$$
y_{1}=y_{i}-y_{m}
$$

122

$$
z_{1}=z_{i}-z_{m}
$$

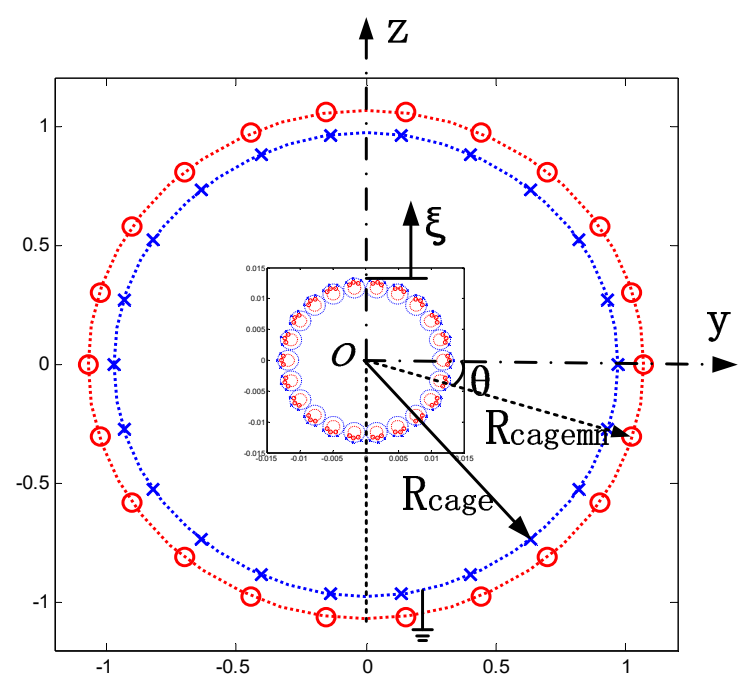

(a)

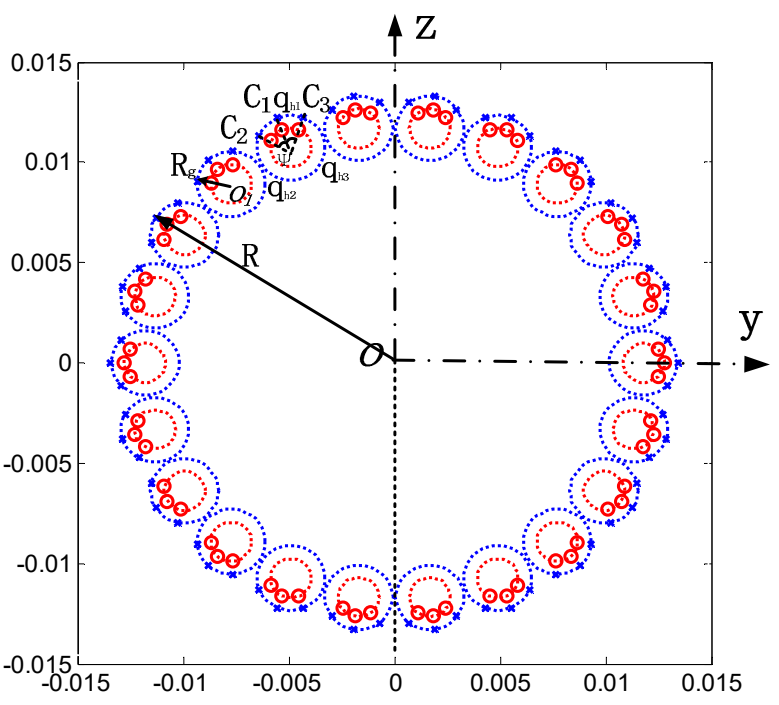

(b)

Figure 4.The location of helical charges and boundary points in the cross section.(a) Arrangement of simulation helical charges and boundary on the corona cage and conductor; (b) Arrangement of simulation helical charges and boundary on the conductor. 


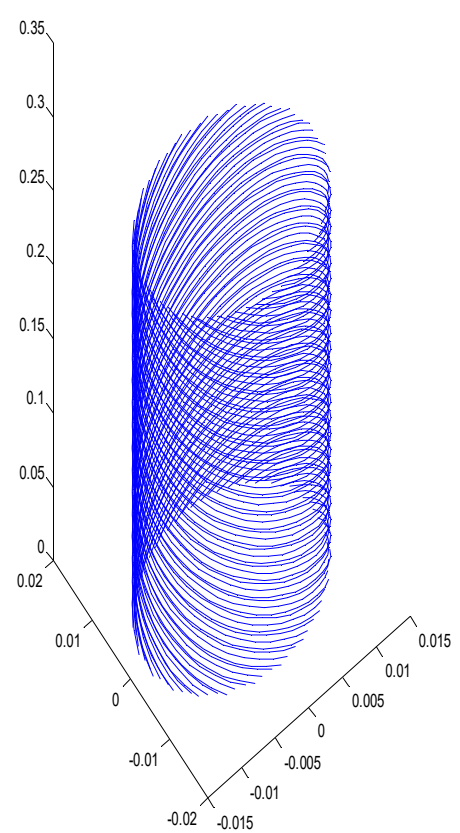

(a)

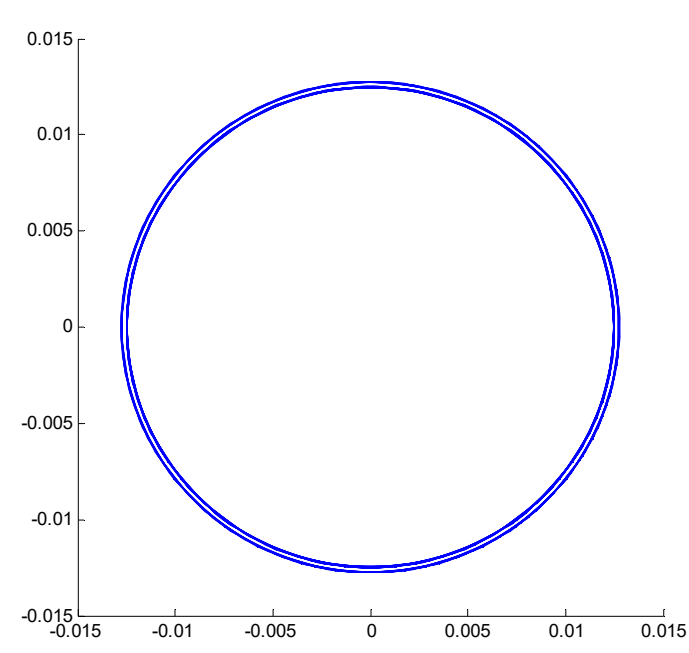

(b)

Figure4. Helical charges' arrangement of with Pitcho on the conductor (a) Main view of helical charges' arrangement (b) Top view of helical charges arrangement

\subsection{Corona onset and charge emission}

According to the Kaptzov assumption, the corona onset charge at different points on the conductor surface is calculated by considering the influences of space charge. Supposing that voltage $U_{t}=U_{\text {app }} \cdot \sin (\omega \cdot t)$ is applied at moment $t$, then,

$$
P_{\text {cond }} Q_{\text {cond }}+P_{\text {space }} Q_{\text {space }}+P_{\text {cage }} Q_{\text {cage }}=U_{t}
$$

$$
P_{\text {cond } 1} Q_{\text {cond }}+P_{\text {space } 1} Q_{\text {space }}+P_{\text {cagel } 1} Q_{\text {cage }}=0
$$

$$
E_{x}=E_{x 1} \cos \delta \cos \beta+E_{y 1} \sin \beta+E_{z 1} \sin \delta \cos \beta
$$

$$
E_{y}=E_{x 1} \cos \delta \sin \beta+E_{y 1} \cos \beta+E_{z 1} \sin \delta \sin \beta
$$

$$
E_{z}=E_{x 1} \sin \delta+E_{z 1} \cos \delta
$$

Where,

$$
\begin{aligned}
& E_{x 1}=f x_{\text {cond }} Q_{\text {cond }}+f x_{\text {space }} Q_{\text {space }}+f x_{\text {cage }} Q_{\text {cage }} \\
& E_{y 1}=f y_{\text {cond }} Q_{\text {cond }}+f y_{\text {space }} Q_{\text {space }}+f y_{\text {cage }} Q_{\text {cage }} \\
& E_{z 1}=f z_{\text {cond }} Q_{\text {cond }}+f z_{\text {space }} Q_{\text {space }}+f z_{\text {cage }} Q_{\text {cage }}
\end{aligned}
$$

In (13), (14), (18) and (19), $Q_{\text {cond }}, Q_{\text {space }}$ and $Q_{\text {cage }}$ represent the vector of simulation line charges of the conductor, space and cage walls, respectively. 
143

144

145

146

147

148

149

150

In (13), $P_{\text {cond }}, P_{\text {space }}$ and $P_{\text {cage }}$ are the potential coefficient matrix of $Q_{\text {cond }}, Q_{\text {space }}$ and $Q_{\text {cage }}$ to the charge emitting points on the surface of the conductor, respectively.

In (14), $P_{\text {cond } 1,} P_{\text {space } 1}$ and $P_{\text {cage } 1}$ are the potential coefficient matrix of $Q_{\text {cond }}, Q_{\text {space }}$ and $Q_{\text {cage }}$ to the boundary points on corona cage wall, respectively.

Equating the applied voltage with calculated potential at boundary points, the unknown simulation charges can be determined through (13) and (14).

In (15)-(17), $\delta$ and $\beta$ are the inclination angles vector of line charges having length $l$ on the $x-y$ and $x-z$ planes, as demonstrated in Figure 5.

Figure 5.theinclination angles of linear charges on thex-y and $x-z$ planes

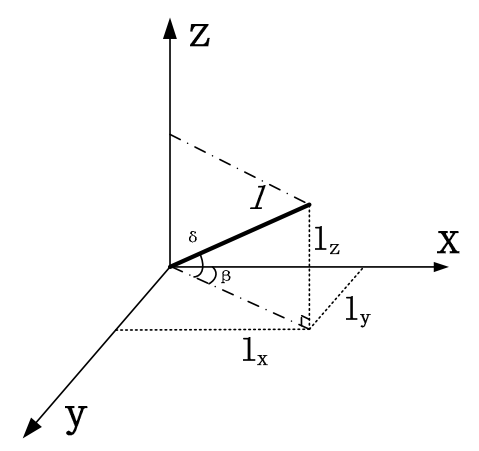

In (18), $f x_{\text {cond }}, f x_{\text {space }}$ and $f x_{\text {cage }}$ are the field strength coefficients matrix of $Q_{\text {cond }}, Q_{\text {space }}$ and $Q_{\text {cage }}$ to the emitting points on the conductor surface at the $x$ direction, respectively.

In (19), fycond, $f y_{\text {space }}$ and $f y_{\text {cage }}$ are the field strength coefficients matrix of $Q_{\text {cond, }}, Q_{\text {space }}$ and $Q_{\text {cage }}$ to the emitting points on the conductor surface at the y direction, respectively.

In (20), fzcond, $f z_{\text {space }}$ and $f z_{\text {cage }}$ are the field strength coefficients matrix of $Q_{\text {cond }}, Q_{\text {space }}$ and $Q_{\text {cage }}$ to the emitting points on the conductor surface at the $z$ direction, respectively.

The corona onset electric field strength $E_{o n}$ is calculated using Peek's formula. When the electric field strength on the conductor surface is $E_{\text {on }}$ in (21), the vector of conductor charge is defined as the corona onset charge $Q_{c r i t}$ and $Q_{c r i}$, which is used as the criteria.

$$
E_{\text {on }}=\left|E_{x}+E_{y}+E_{z}\right|
$$

At moment $t$, comparing $Q_{\text {cond }}$ with the corona onset criteria $Q_{c r i t}$ or $Q_{c r i-}$ to judge whether points on the conductor surface are corona onset. For example, for the $r_{\text {th }}$ simulation line charge point of the conductor, the $r_{\text {th }}$ element of $Q_{\text {cond }}$ is selected for comparison with the $r_{\text {th }}$ element of $Q_{\text {crit }}$ or $Q_{\text {cri. }}$. If

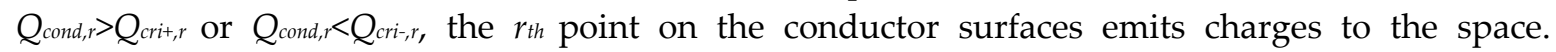
Supposing that the emitting charge vector on the surface of the conductor is $Q_{e m i}$, the $r_{\text {th }}$ element is $Q_{e m i, r}=Q_{c o n d, r}-Q_{c r i t, r}$ or $Q_{e m i, r}=Q_{c o n d, r}-Q_{c r i, r}$ and the $Q_{c o n d, r}$ changes into $Q_{c r i t, r}$ or $Q_{c r i, r}$.

\subsection{Charge migration and recombination}

When the polarity of the space line charge is same or different from that of the conductor, the space line charge will be pushed away from or attracted to the conductor. In the calculation model, within a time interval $\Delta t$, the displacement of space line charge $\Delta d x$ at the $x$ direction is

$$
\Delta d_{x}=\mu \cdot E_{x} \cdot \Delta t
$$

The displacement of space line charge $\Delta d_{y}$ at the $y$ direction is 
175

176

177

178

179

180

$$
\Delta d_{y}=\mu \cdot E_{y} \cdot \Delta t
$$

The displacement of space line charge $\Delta d z$ at the $z$ direction is

$$
\Delta d_{z}=\mu \cdot E_{z} \cdot \Delta t
$$

Where, $\mu$ is the ion mobility. $E_{x}, E_{y}$ and $E_{z}$ represent the electric field strength at the $x, y$ and $z$ directions, respectively.

Due to the recombination of positive and negative charges, the charge quatity gradually reduces, the charge density decreases as time passes. During $\Delta t$, at the beginning, the ion space charge density is

$$
n_{i 0}=\frac{\left|q_{i 0}\right|}{e \cdot \Delta v_{i}}
$$

In (25), $e$ is the quantity of electronic charges, $e=1.6 \times 10^{-19} \mathrm{C}$, $q_{i 0}$ is the $i_{t h}$ space line charge quantity, and $\Delta v_{i}$ is the spherical control volume of $q_{i 0}$.

Therefore, after time $\Delta t$, the $i$ th space line charge changes to be

$$
q_{i}=\frac{q_{i 0}}{1+n_{i 0} \cdot \Upsilon \cdot \Delta t}
$$

In (26), the recombination coefficient $Y$ is $1.5 \times 10^{-12} \mathrm{~m}^{3} / \mathrm{s}$.

\subsection{Calculation of corona loss and corona current}

The space line charge moves back and forth in the AC electric field and the consumed energy is corona loss. When the $i_{\text {th }}$ space line charge moves $\Delta d_{i x}, \Delta d_{i y}$ and $\Delta d_{i z}$ at the $x, y$ and $z$ directions, the corona loss is

$$
W_{i}=q_{i} \cdot E_{i x} \cdot \Delta d_{i x}+q_{i} \cdot E_{i y} \cdot \Delta d_{i y}+q_{i} \cdot E_{i z} \cdot \Delta d_{i z}
$$

The corona power loss in a frequency term is

$$
W=\sum_{\text {cycle }} \sum_{i=1}^{N_{s c}} W_{i}
$$

In (28), $N_{s c}$ indicates the number of space line charge in a time step. And cycle is the time step quatity in a term. The power of corona loss of the conductor in unit length is

$$
P=f \cdot \frac{W}{l_{\text {cond }}}
$$

In (29), $f$ and $l_{\text {cond }}$ represent the power frequency and conductor length, respectively, $f=50 \mathrm{~Hz}$.

The total corona current $I_{c o r}$ consists of displacement current $I_{\text {disp }}$ and conduction current Icond, Idisp can be expressed by the variable quantity of simulation charge on conductor surface, but the capacitive current $I_{\text {cap }}$ before the corona onset must be drawn from the Idisp. And Icond is related to the movement of the ion space charge.

$$
I_{\text {cor }}=\left(I_{\text {disp }}-I_{\text {cap }}+I_{\text {cond }}\right) / l_{\text {cond }}
$$

Where, 


$$
I_{\text {disp }}=\frac{\Delta \sum_{N_{1}} q_{\text {cond }}}{\Delta t}
$$

$$
I_{\text {cap }}=\frac{\Delta \sum_{N_{1}} q_{\text {cond }, \text { uncor }}}{\Delta t}
$$

$$
I_{\text {conv }}=\sum_{N} \frac{q_{\text {space }} \mu E_{s}^{2}}{u_{t}}
$$

In (30)-(32), q cond is the simulation charge of the conductor considering the corona discharge, $q_{\text {cond, uncor }}$ is the simulation charge of the conductor ignoring the corona discharge. $N_{1}$ is the quantity of $q_{\text {cond }}, q_{\text {space }}$ is the charge quantity of space line charge, $E_{s}$ is the electric field at $q_{\text {space }}$ 's location, and $u t$ is the applied voltage at the moment $t$.

\section{Corona-loss calculation and analysis of single stranded conductor in the small corona cage}

Literature [19] presents the test results of corona loss of a single stranded conductor LGJ-300/40 and LGJ-400/35 in a small corona cage with a cross-section dimension of $1.8 \mathrm{~m}$. The total length and the measuring section length are $4 \mathrm{~m}$ and $3 \mathrm{~m}$, respectively, and the protection section length in both sides is $0.5 \mathrm{~m}$. Due to the test ambient atmosphere pressure, temperature and humidity were 101.15 $\mathrm{kPa}, 25.3^{\circ} \mathrm{C}$ and $29.8 \%$, the positive ion motility $\mu_{+}$and negative ion motility $\mu_{\text {- }}$ are selected, $\mu_{+}=1.5 \times 10^{-4} \mathrm{~m}^{2} /(\mathrm{V} \cdot \mathrm{s}), \mu_{-}=1.92 \times 10^{-4} \mathrm{~m}^{2} /(\mathrm{V} \cdot \mathrm{s})[22]$. By setting the pitch factor $P F$ and surface roughness coefficient $m$ to be 11 and 0.78 for LGJ-300/40, 11 and 0.83 for LGJ-400/35, and assuming that the conductor length calculated is $3 \mathrm{~m}$. Each power cycle has been divided into 200 steps which represent a good compromise between the accuracy and the computational time, the changes of corona loss with the increase of test voltages was studied, as shown in Figure 6, and comparison between the measured results and calculated values is shown in Table 2 and Table 3 . It can be seen that the calculated values agreed satisfactorily with the measured values, but the calculation error is larger at the corona onset point, one possible reason giving rise to the result described above is: the actual conductor surface roughness is non-uniform, the random electric discharge firstly appears at several points on the conductor surface with the rise of voltages, but the overall corona onset on the conductor surface will occur only when the applied voltage increases to a certain degree. Therefore, the 3-D corona-loss calculation model can reflect the actual physical process of corona discharge.

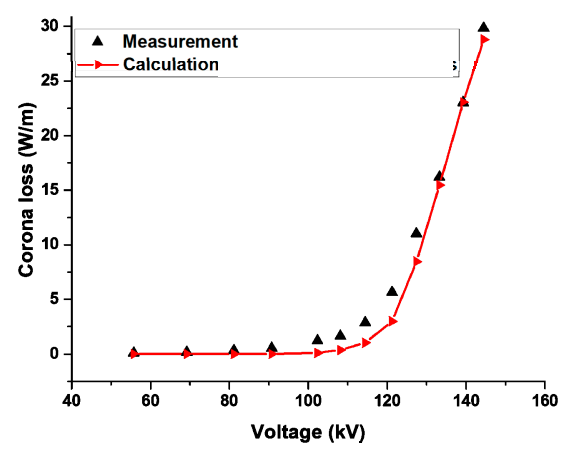

(a)

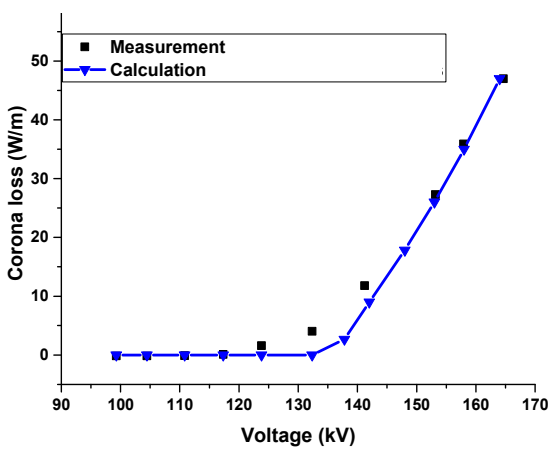

(b)

231 Figure 6.Calculation and measurement results of conductors corona loss:(a)LGJ300-40; (b)LGJ400-35

Table2. Relative error between calculation and measurement results for LGJ300-40

\begin{tabular}{llll}
\hline Voltage applied(kV) & Test values $(\mathrm{W} / \mathrm{m})$ & calculated values $(\mathrm{W} / \mathrm{m})$ & error $(\%)$
\end{tabular}




\begin{tabular}{cccc}
114.4 & 2.87 & 1.03 & -64.22 \\
127.42 & 11.03 & 9.60 & 12.9 \\
133.26 & 16.20 & 15.46 & 4.57 \\
139.27 & 23.03 & 23.06 & -0.11 \\
144.36 & 29.84 & 28.79 & 3.50 \\
\hline
\end{tabular}

Table3. Relative error between calculation and measurement results for LGJ400-35

\begin{tabular}{cccc}
\hline Voltage applied(kV) & Test values (W/m) & calculated values (W/m) & error (\%) \\
\hline 123.79 & 1.59 & 0 & 100 \\
141.23 & 11.32 & 9.68 & 14.49 \\
153.15 & 27.27 & 26 & 4.66 \\
157.90 & 35.9 & 35 & 2.51
\end{tabular}

For LGJ300-40, Uapp $=140 \mathrm{kV}$. Figure 7 shows how the single-conductor corona loss changes with the cycle no., the steady state solution is achieved after three cycles and the corona loss value did not change significantly from one cycle to another. The corona current waveform is shown in Figure 8. The motion trajectories of space line charges corresponding to different instants of the voltage cycle are shown in Figure 9.

The cycle begins at point $a$, with the applied voltage $U_{t}=0$ and starting to increase in the positive half-cycle, a mass of negative space line charge created in the preceding negative half cycle is located at some distance away from the conductor, as shown in Figure 9(f). From point $a$ to $b$, with the positive voltage increases, the electric field surrounding the conductor also increases, so the negative space line charges move back to the conductor at increasing speed. At point $b$, the conductor surface electric field equals $E_{o n}$, thereafter, positive corona discharge occurs on the conductor and the positive ions generated move away from the conductor, while the electrons are neutralized on the contact with conductor, the space line charge situation is depicted in Figure 9(a). The positive corona discharge continues till point $c$, the corona discharge disappears, considering that the conductor surface electric field is reduced by the mass of positive space line charges surrounding the conductor, the voltage of the corona activity quenching is higher than that corresponding to corona onset, as shown in Figure 9(b). the positive space line charge reaches the largest distances at the point $d$. and the situation in negative half cycle is similar to the positive half cycle except for the polarity's changing, the negative corona starts at point $e$ and ends at point $f$, the space line charges trajectories is shown in Figure 9 (d) and (e). 


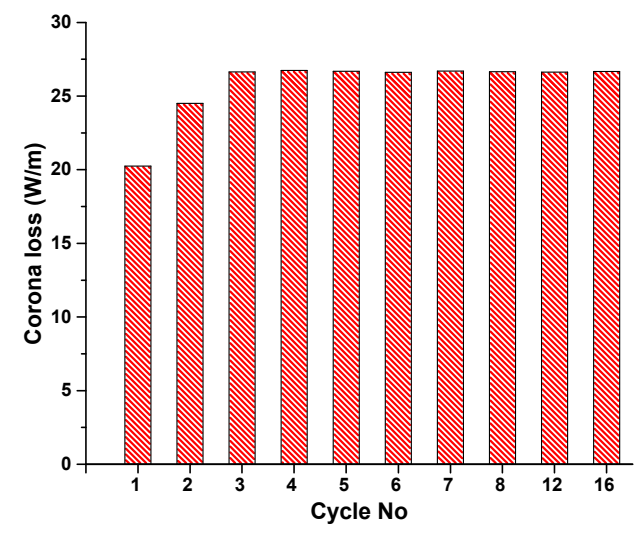

Figure7. Variation of corona-loss calculation results in the 16 cycles

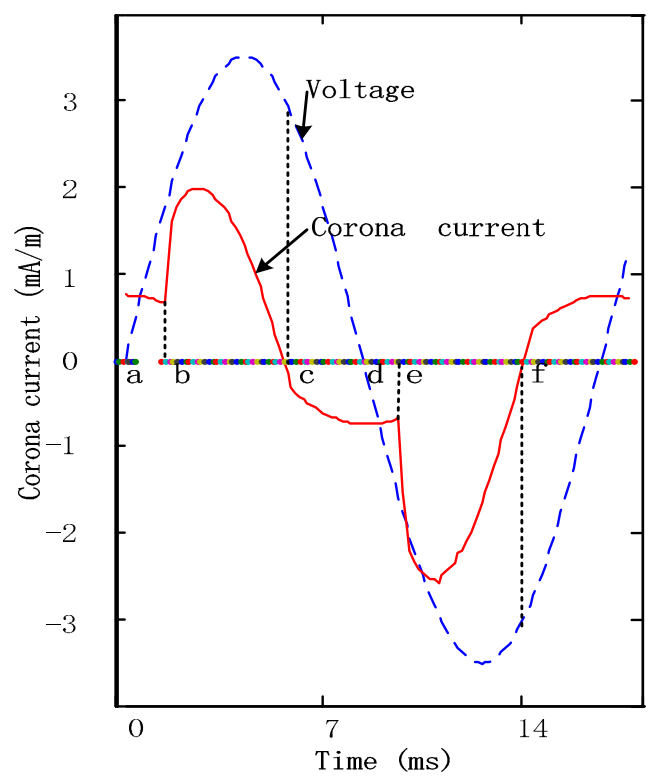

259

Figure 8. Corona current and voltage waveforms in power cycle

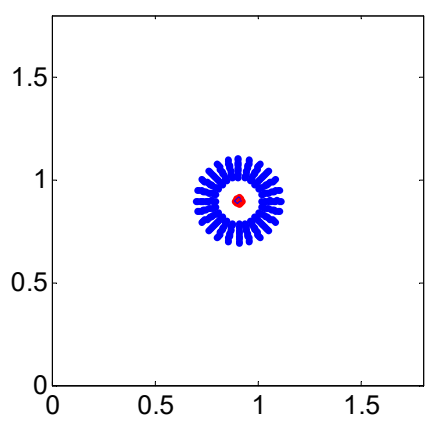

(a)

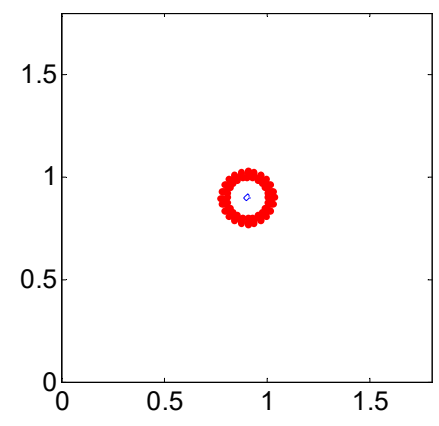

(b)

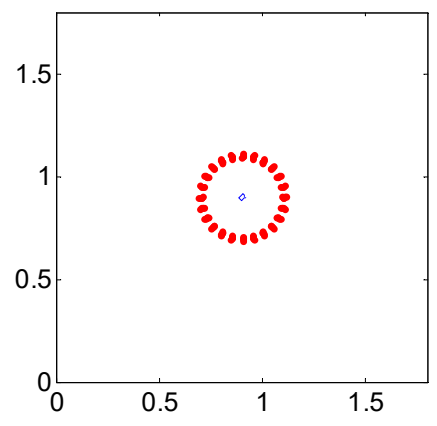

(c) 


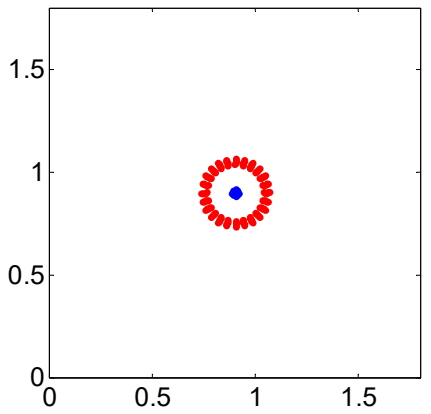

(d)

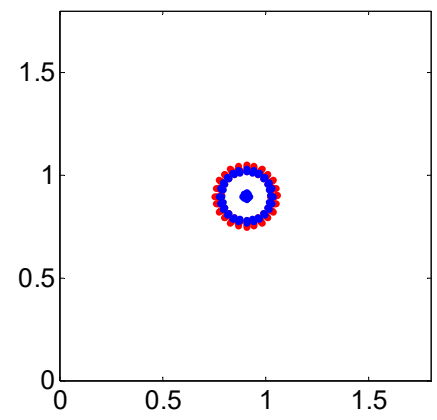

(e)

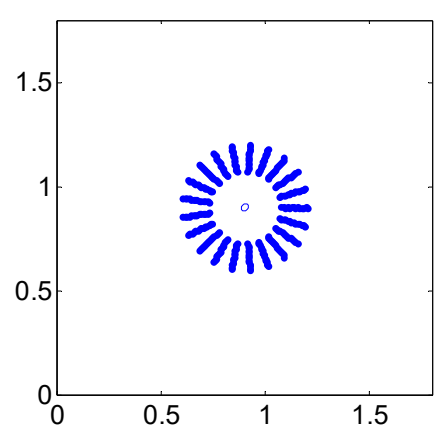

(f)

Figure 9. Space line charge trajectories above corona onset: (a) Positive corona onset; (b) Positive ions moving away; (c) Positive ions return; (d) Negative corona onset; (e) Positive and negative ions moving on the contrary; (f) Negative ions return.

\section{Corona-loss analysis of bundle-sag conductors in the UHV corona cage}

The calculation model proposed in this paper can also analyze the corona characteristics of the conductor which has sag. In figure 10, the UHV corona cage set in Ping'an County, Xining City, China (elevation 2,200 m), with a square cross-section $(8 \mathrm{~m} \times 8 \mathrm{~m}$ ) and a total length of $35 \mathrm{~m}$ was used. The corona cage is composed of a $25 \mathrm{~m}$ long measuring section and two protective sections on each side of the measuring section, being $5 \mathrm{~m}$ long respectively. Based on the self-developed integrated photoelectric corona loss measurement system, the test was carried out for bundle conductors $4 \times$ LGJ720 on a calm day without wind, the bundle spacing is $450 \mathrm{~mm}$,the equivalent roughness coefficient value $m$ is adopted as $0.75, P F$ is selected as $10, R=18.12 \mathrm{~mm}, R_{g}=4.529 \mathrm{~mm}$. The test ambient atmosphere pressure, temperature and relative humidity were $78.5-78.8 \mathrm{kPa}$, $10.2-15.3^{\circ} \mathrm{C}$ and $68.8-82.8 \%$, so $\mu$ - and $\mu$ - are selected as $1.32 \times 10^{-4} \mathrm{~m}^{2} /(\mathrm{V} \cdot \mathrm{s})$ and $1.65 \times 10^{-4} \mathrm{~m}^{2} /(\mathrm{V} \cdot \mathrm{s})$ respectively[22]. The diagrammatic sketch of conductors with sag in calculation model on front view is shown in Figure 11, the changes trend of conductors at the axial direction is approximate to the parabola in corona cage, coordinates matrix of bundle conductors with sag can be obtained by the coordinate matrix's shifting transformation and rotation transformation from long and straight conductors by (33).

$$
[B]=\left[\begin{array}{l}
X_{1} \\
Y_{1} \\
Z_{1}
\end{array}\right]=[A]\left[M_{1}\right]\left[M_{2}\right]=\left[\begin{array}{l}
X \\
Y \\
Z
\end{array}\right]\left[M_{1}\right]\left[M_{2}\right]
$$

In (33), $B$ is coordinates matrix of bundle conductors with sag, $A$ is coordinates matrix of long and straight conductors, $M_{1}$ is matrix of shifting transformation, $M_{2}$ is matrix of rotation transformation.

And the conductors with sag between $0.15 \mathrm{~m}$ and $0.25 \mathrm{~m}$ is calculated. As is shown in Figure 12, test results are compared with calculation results. It can be seen that the corona losses calculated around the vicinity of corona onset point have significant differences on different conductors' sag condition, however, as the applied voltage increases, the corona loss has the trend of closing to the 

one certain value. And the conductors with sag $=0.2 \mathrm{~m}$ can approximate the practical situation more 288 accurately.

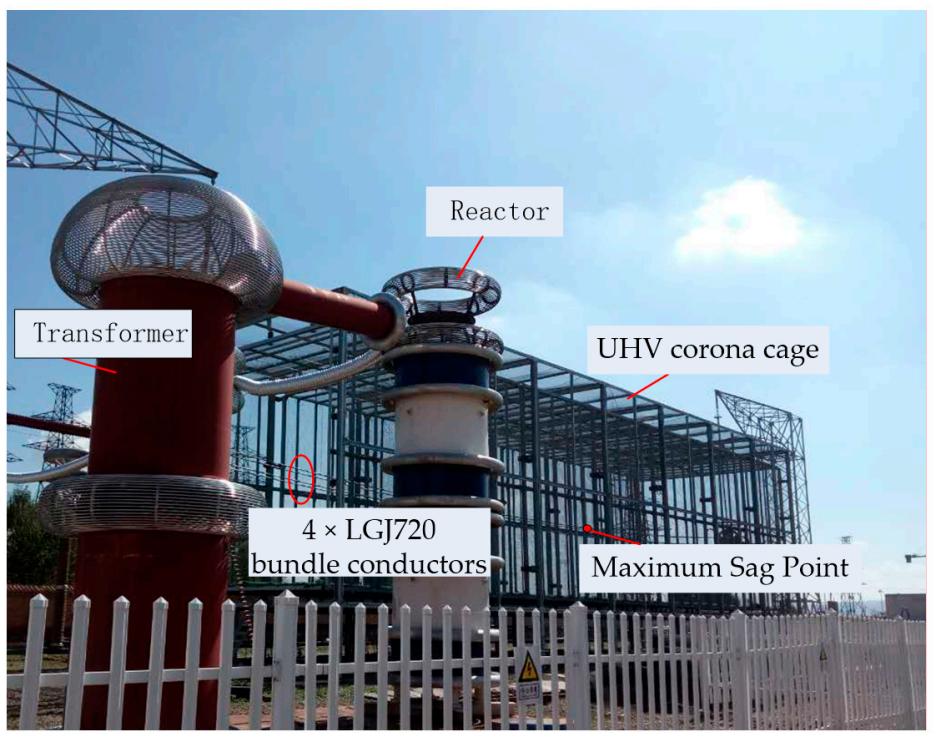

Figure 10. UHV corona cage at Ping'an

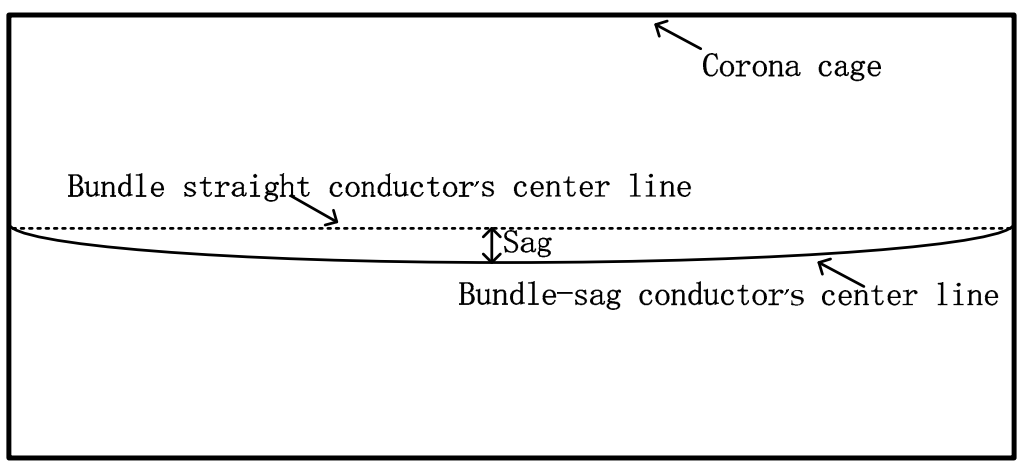

Figure 11. The conductor with sag in calculation model on front view

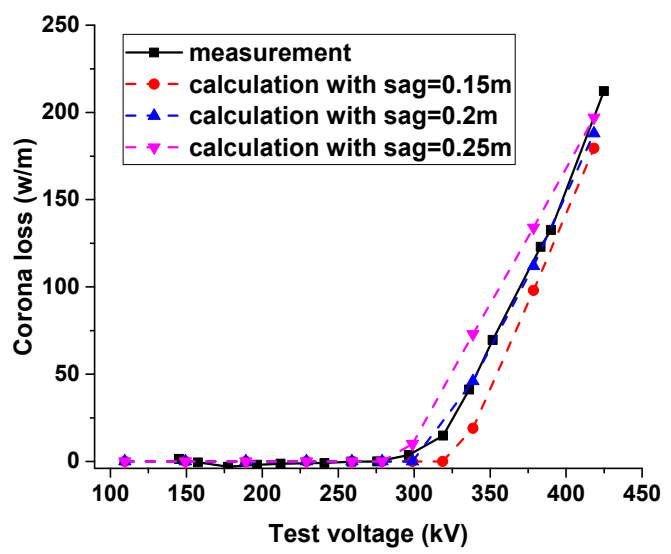

Figure 12. Corona losses with different sag of conductor 
Based on helical charges, the Kaptzov assumption is used and the Deutsch assumption is abandoned, and the inhomogeneity of space line charge emission and the actual structures of conductor are taken into consideration. By introducing the measuring value of ion mobility and simulating the processes including emission, transfer and recombination of space line charges, the calculation method for 3-D corona loss of the conductor in the corona cage is established.

The test results of corona loss well coincide with the calculation results on single stranded conductor and bundle-sag conductors in different corona cages. Compared with 2-D corona loss calculation models, the model in this paper can take the electric-field nonuniformity along the axial direction on the conductor surface, it is more accurate to analyze physical process of corona discharge.

Acknowledgments: The authors would like to thank the reviewers of this paper for the useful comments. This work is supported by National Natural Science Foundation of China (51577069) and the National Grid Corporation of Science and Technology (Grant no. SGTYHT/15-JS-191).

Author Contributions: Shilong Huang established the 3-D calculation model for corona loss; Shilong Huang analyzed the data; Yunpeng Liu, Shugang Liu, Daran Liu and Zhicheng Huang contributed reagents/materials/analysis tools; Shilong Huang and Daran Liu wrote the paper.

Conflicts of Interest: The authors declare no conflict of interest.

\section{References}

1. Liu, Z.Y. Ultra-high voltage grid; China Economic Press: Beijing, China, 2005.

2. Zhang G.Z.; Cheng G.S.; Wan B.Q. Study on EM environment of UHV test line segment. High Voltage Engineering. 2008, 34, 438-441.

3. Liu, Z.Y. Global Energy Internet; China Economic Press: Beijing, China, 2015.

4. Maruvada P S. Corona performance of high-voltage transmission lines; Research Studies Press Ltd: London, 2000.

5. Frans J.S.; Andrew M; Klas R. Evaluation, verification and operational supervision of corona losses in Sweden. IEEE Trans on Power Delievery. 2007, 22, 1210-1217.

6. Anderson J.G.; Zaffanella L.E. Project UHV Test Line Research on the Corona Performance of a Bundle Conductor at $1000 \mathrm{kV}$. IEEE Transactions on PAS.1970, 89, 1168-1178.

7. Vinh T.; Shih C.H.; King J.V.; Roy W.R. Audible Noise and Corona Loss Performance of 9-Conductor Bundle for UHV Transmission Lines. IEEE Transactions on PAS, 1985,104, 2764-2770.

8. Juette G.W.; Zaffanella L.E. Radio Noise, Audible Noise, and Corona Loss of EHV and UHV Transmission Lines Under Rain: Predetermination Based on Cage Tests. IEEE Transactions on PAS, 1970, 89, 1168-1178.

9. Kolcio N.; Caleca V.; Marmaroff S.J.; Gregory W.L. Radio-Influence and Corona-Loss Aspects of AEP 765-kV Lines. IEEE Transactions on PAS, 1969, 88, 1343-1355.

10. Chartier V.L.; Shankie D.F.; Kolcio N. The Apple Grove 750-kV Project: Statistical Analysis of Radio Influence and Corona-Loss Performance of Conductors at $775 \mathrm{kV}$. IEEE Transactions on PAS, 1970, 89, 867-881.

11. Liu Y.P.; You S.H.; Wan Q.F. Design and realization of AC UHV corona loss monitoring system. High Voltage Engineering, 2008, 34, 1797-1801.

12. Liu Y.P.; You S.H.; Wan Q.F. Research on UHV AC single circuit test line corona losses under rain. Proceedings of the CSEE, 2010, 30, 114-119.

13. Clade J. J.; Gary C. H.; Lefevre C.A. Calculation of corona losses beyond the critical gradient in alternating voltage. IEEE Trans on PAS,1969, 88,695-703. 
14. Clade J. J.; Gary C. H. Predetermination of corona losses under rain: experimental interpreting and checking of a method to calculate corona losses. IEEE Trans on PAS, 1970, 89,853-860.

15. Clade J. J.; Gary C. H. Predetermination of corona losses under rain: influence of rain intensity and utilization of a universal chart. IEEE Trans on PAS. 1970, 89, 1179-1185.

16. Abdel S.M.; Abdel-Aziz E.Z. A charge simulation based method for calculating corona loss on AC power transmission lines. Journal of Physics D: Applied Physics, 1994, 27, 2570.

17. Abdel S.M.; Abdel-Aziz E.Z. Corona power loss determination on multi-phase power transmission lines. Electric power systems research, 2001, 58, 123-132.

18. Li W.; Zhang B.; He J.L. Influence of corona effect on ground level electrical field under EHV/UHV AC transmission lines. High Voltage Engineering, 2008, 34, 2288-2294.

19. YOU S.H.; Lü F.C.; Liu Y.P. AC conductors' corona-loss calculation and analysis in corona cage', Proceedings of the CSEE, 2012, 32, (1), pp.162-170.

20. Lü F.C.; You S. H.; Liu Y. P. AC conductors' corona-loss calculation and analysis in corona cage. IEEE Trans on Power Delivery, 2012, 27, 877-885.

21. Hochberg D.; Edwards G.; Kephart T.W. Representing structural information of helical charge distributions in cylindrical coordinates. Physical Review E.1997, 55, 3765.

22. Liu Y.P.; Huang S.L.; Zhu L. Influence of humidity and air pressure on the ion mobility based on drift tube method. CSEE JPES, 2015, 1, 37-41.

23. Ministry of Machinery Industry of People's Republic of China. Aluminium stranded conductors and aluminium conductors steel-reinforced; Standards Press of China: Beijing, China, 1983. 